\title{
L'agobiopsia renale ecoguidata
}

\author{
N. Campobasso, G. Pannarale, F.P. Schena \\ Cattedra e Divisione di Nefrologia Dialisi Trapianti, Università degli Studi, Policlinico, Bari
}

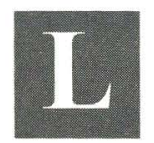

a biopsia renale è considerata oggi un presidio diagnostico di fondamentale importanza per lo studio delle malattie renali, in particolare per le glomerulopatie.

L'approccio percutaneo, introdotto nel 1951 da Iversen e Brun (1), è oggi utilizzato nella stragrande maggioranza delle biopsie, per cui appare evidente che uno dei maggiori problemi nella sua esecuzione è una corretta localizzazione del polo inferiore del rene (2).

Per molti anni è stata utilizzata la tecnica "cieca", basata su punti di repere anatomici, descritta da Kark e Muehrcke nel 1954 (3). Da queste prime esperienze sono state descritte numerose metodiche più o meno complesse e indaginose (Tab. I) con risultati buoni e comunque sovrapponibili: fluoroscopia ad intensificazione d'immagine $(4,5)$, scintigrafia (6), urografia (7) e tomogorafia assiale computerizzata (8). Sebbene con tutte queste metodiche si siano ottenuti prelievi utili per la diagnosi in un'altissima percentuale di casi, molte di esse presentano notevoli limitazioni rispetto ai vantaggi che offrono: problemi di parallasse nelle localizzazioni radiologiche, limitazioni all'uso del mezzo di contrasto nell'insufficienza renale, rapporto costo/beneficio, ecc.

Per tale motivo l'avvento dell'ecografia ha rivoluzionato la metodica bioptica, presentando questa tecnica molti van- taggi e pressocché nessuno svantaggio (Tab. II), ed è diventata oggi la metodica di elezione per la localizzazione del polo renale (9).

Anche per la biopsia ecoguidata sono state descritte numerose metodiche, da quella "a mano libera" (Fig. 1), in cui la discesa dell'ago sul rene viene seguita da una sonda lineare posta trasversalmente al rene e parallelamente all'ago (10), a quella che prevede un sistema di asservimento dell'ago alla sonda (Fig. 2), per-

TAB. I - METODICHE DI LOCALIZZAZIONE

1) Tecnica "cieca" (punti di repere anatomici)

2) Fluoroscopia ad intensificazione di immagine

3) Scintigrafia

4) Pielografia (con piombino sul polo inferiore)

5) Tomografia assiale computerizzata (TAC)

6) Ecografia

\section{TAB. II - VANTAGGI DELL'ECOGRAFIA}

1) Assenza di radiazioni

2) Rapidità, semplicità ed estrema accuratezza con la conseguente ottimizzazione del tempo di ricovero

3) Facile esecuzione, anche al letto del paziente, indipendentemente dal grado di insufficienza renale

4) Visualizzazione continua in "real time" della discesa dell'ago mediante un piccolo punto di contatto cutaneo 
mettendo un controllo dinamico del bersaglio nonché la strettta sorveglianza degli spostamenti del rene durante gli atti respiratori (11).

Nella nostra Divisione già da alcuni anni la biopsia renale viene eseguita sotto guida ecografica (12) utilizzando il protocollo qui descritto (Tab. III).

Il paziente, ricoverato almeno un giorno prima della biopsia, viene sottoposto ad una serie di indagini atte ad escludere una diatesi emorragica. Al momento della biopsia, dopo blanda sedazione, viene posto in posizione prona e sottoposto ad esame ecografico preliminare. In questa fase viene localizzato con precisione il polo inferiore del rene sui piani cutanei e definito il rene nei suoi limiti, nella sua forma e nella sua profondità, utilizzando una sonda macroconvex (3.5 MHz). In assenza di controindicazioni specifiche la sede scelta per la puntura è il polo inferiore del rene sinistro. La precisione nella scelta del punto di infissione dell'ago è agevolata dalla possibilità di far comparire sul monitor la traccia della presunta traiettoria dell'ago. Dopo disinfezione ed anestesia locale, la cute viene incisa in corrispondenza della proiezione cutanea del polo renale. Adattata alla sonda una guida per l'ago da biopsia, questo viene fatto procedere attraverso i piani cutanei e muscolari fino alla capsula renale, visualizzandolo costantemente sul monitor (Fig. 3). Se ne può, quindi, in ogni momento modificare la traiettoria in modo da pungere il rene inferiormente all'ultima piramide. Superata con la punta dell'ago la capsula renale, si esegue il prelievo. L'ago da noi utilizzato è il Tru Cut (Travenol). È nostra consuetudine eseguire due prelievi in modo da avere a disposizione materiale sufficiente per la microscopia

\section{TAB. III - METODICA}

1) Ecografia preliminare per la localizzazione del polo inferiore

2) Disinfezione, anestesia locale ed incisione della cute

3) Avanzamento dell'ago (attraverso la guida) sotto continua visualizzazione ecografica, fino a penetrare con la punta la capsula al polo inferiore

4) Sganciamento della sonda dall'ago e manovra di prelievo

5) Ripetizione della procedura per il prelievo di un secondo frustolo
Fig. 1 - Biopsia ecoguidata "a mano libera": viene seguita la discesa dell'ago sul polo renale tenendo la sonda lineare sulla linea ascellare.

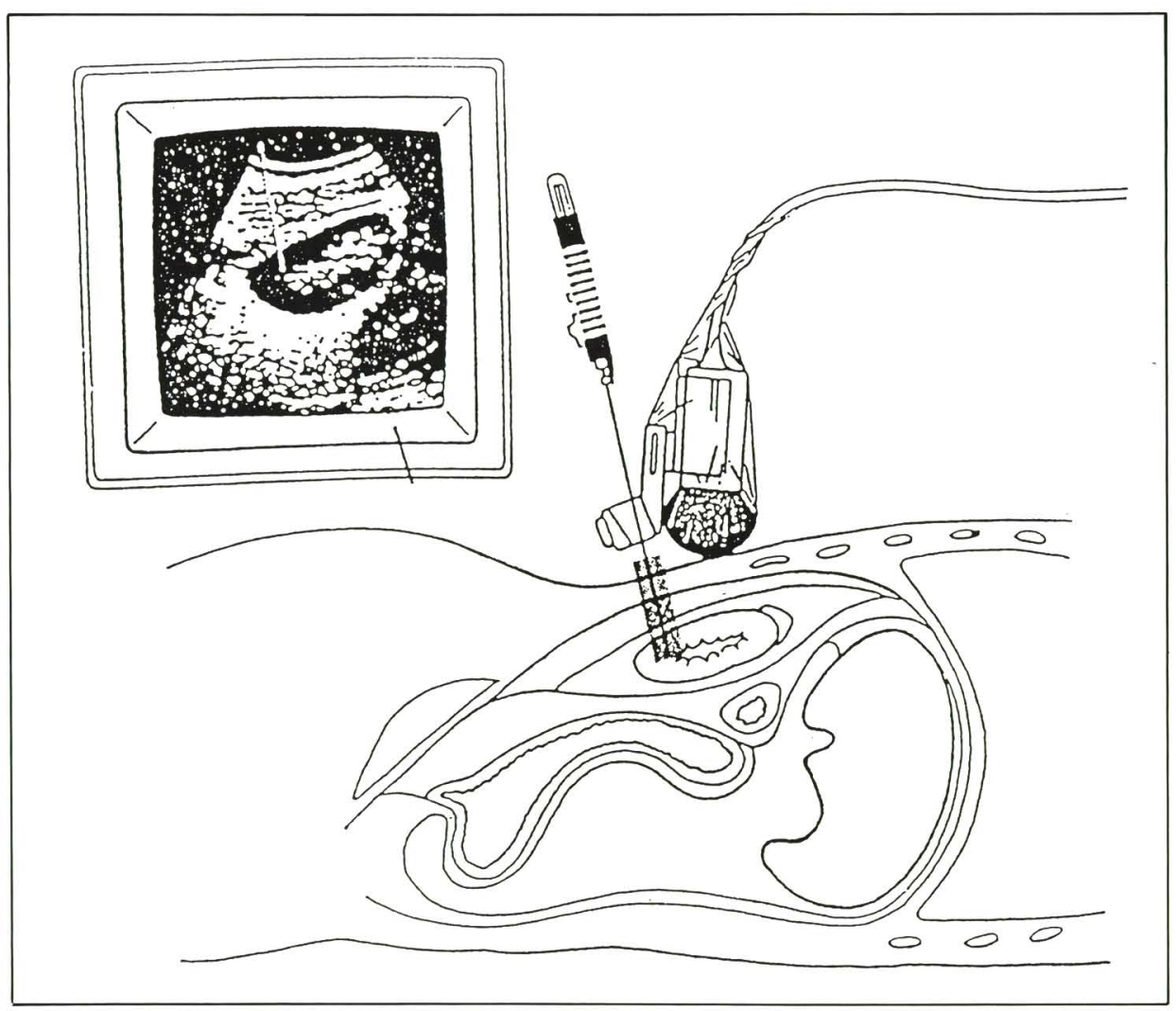

Fig. 2 - Biopsia ecoguidata mediante sistema di asservimento dell'ago alla sonda. 


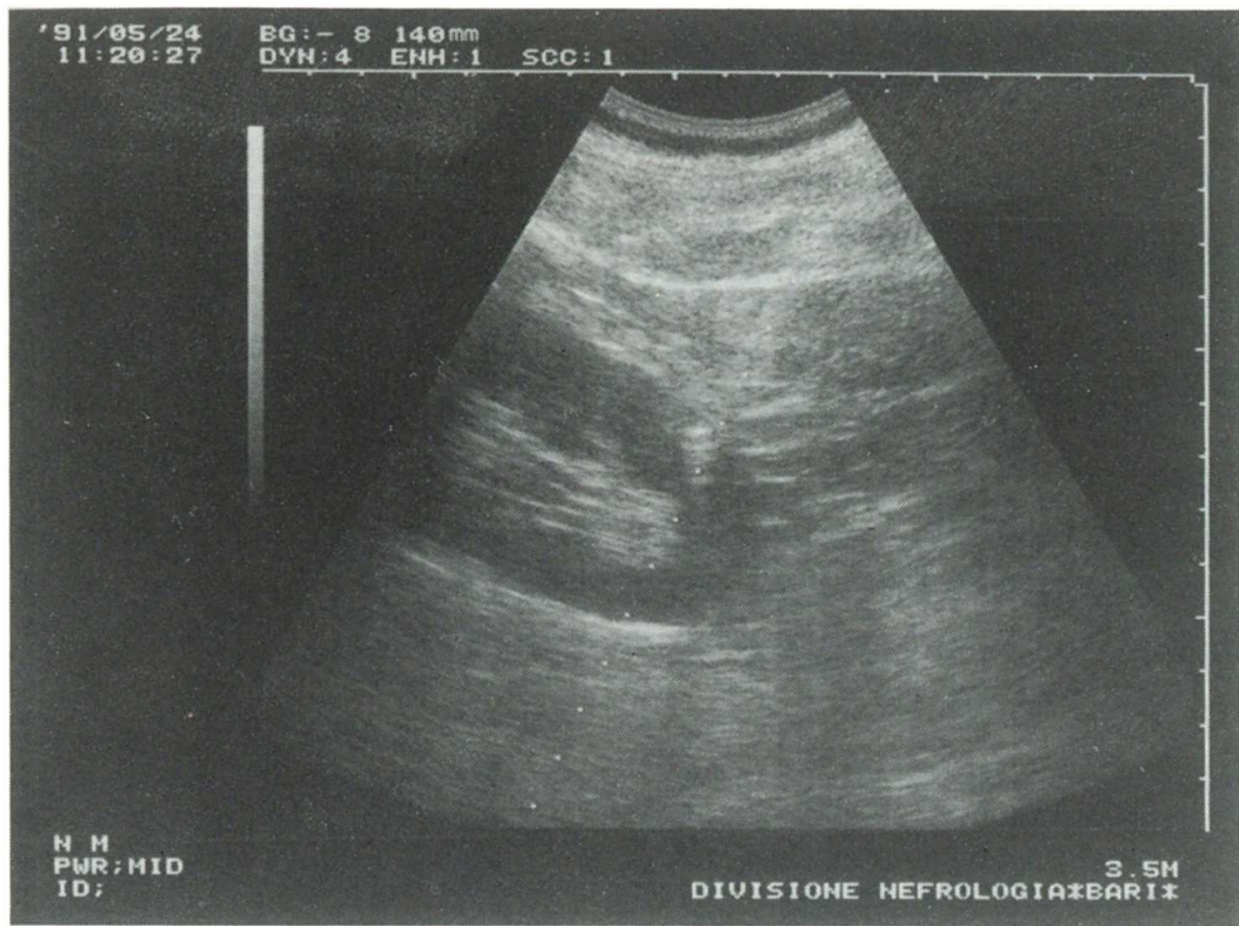

Fig. 3 - Visualizzazione ecografica della penetrazione dell'ago bioptico nel parenchima renale.

ottica, l'immunofluorescenza ed eventualmente la microscopia elettronica. Immediatamente dopo il prelievo il campione bioptico viene esaminato al microscopio a piccolo ingrandimento per verificare l'adeguatezza del frustolo. Il rene biopsiato viene riesaminato ecograficamente nelle ore successive con lo scopo di evidenziare l'eventuale formazione di ematomi perirenali. Una ulteriore ecografia renale viene inoltre effettuata a distanza di 24 e 48 ore dalla biopsia (Fig. 4).

Con tale metodica, dal maggio ' 90 al maggio ' 93 , sono state eseguite presso la nostra Divisione 343 biopsie renali consecutive, ricavandone materiale utile per la diagnosi nel $98 \%$ dei casi. Per ciascuna seduta bioptica sono stati eseguiti all'incirca tre affondamenti, che hanno dato esito a frustoli contenenti una media di 18 glomeruli.

La procedura è generalmente portata $\mathrm{a}$ termine in un tempo complessivo di venti minuti circa ed è sempre stata ben tollerata da tutti i pazienti nonostante un ampio ambito di variabilità dell'età molto ampio compreso tra 14 e 75 anni. In 30 pazienti il controllo ecografico post-bioptico ha evidenziato la presenza di un ematoma perirenale, rilevante dal punto di vista clinico solo in due casi, ore dalla biopsia. emotrasfusioni.

Molto più alta, pari al 30\%, è stata l'incidenza di macroematuria, peraltro transitoria ed asintomatica.

In nessun caso sono state rilevate complicanze tardive post-bioptiche.

Le nostre percentuali di successi e complicanze sono analoghe alle recenti ed ampie esperienze riportate in letteratura $(13,14)$; tuttavia nella nostra casistica, in contrasto con i dati di Burstein (14), la maggiore incidenza di complicanze post-bioptiche si è verificata nei pazienti a rischio per l'età avanzata, la patologia di base (amiloidosi, grave danno vascolare, insufficienza renale avanzata) e le turbe dell'emostasi.

L'incidenza di complicanze nelle biopsie ecoguidate non differisce sostanzialmente da quella riportata in era preecografica (15). Tuttavia noi riteniamo che un diminuito numero di complicanze, ottenuto con l'introduzione della biopsia ecoguidata, sia stato paradossalmente compensato nelle casistiche da un'aumentata capacità di diagnosticarle, dovuto all'uso sistematico dell'ecografia di controllo nell'immediato periodo post-bioptico, che ne permette inoltre un preciso monitoraggio nel tempo.

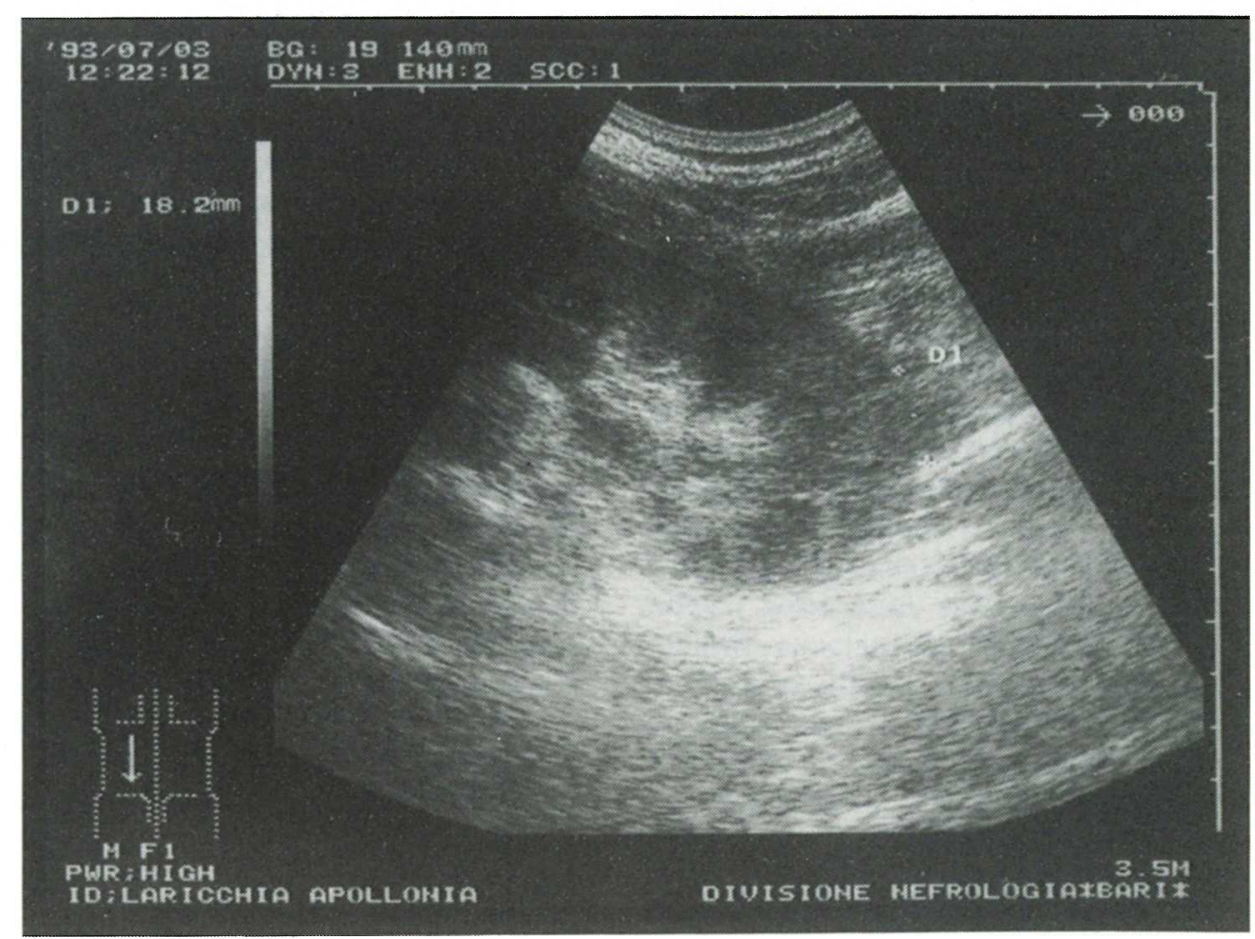

Fig. 4 - Piccolo ematoma perirenale asintomatico al polo inferiore evidenziato dall'ecografia di controllo a 24 
A convalida di questa affermazione ci sono le casistiche degli Autori (16 - 18) che hanno valutato l'incidenza di ematomi post-bioptici con metodiche più sofisticate e più precise dell'ecografia, come la tomografia assiale computerizzata. Essi hanno rilevato raccolte perirenali in una percentuale variabile tra il 57 e l' $85 \%$ dei pazienti sottoposti a biopsia. Sia questi Autori che i nostri dati indicano tuttavia che gli ematomi si risolvono spontaneamente nel giro di tre mesi, raramente si infettano e non richiedono terapia antibiotica parenterale né drenaggio chirurgico.

In conclusione riteniamo che l'introduzione degli ultrasuoni abbia determinato un notevole progresso nella pratica bioptica nefrologica. L'ecografia ha reso più agevole e rapida l'esecuzione della biopsia, eliminando gli esami preparatori radiologici. La tecnica bioptica è risultata più affidabile, perché ha permesso una più precisa localizzazione del polo renale, una visualizzazione diretta della biopsia ed una più alta percentuale di successi con una maggiore adeguatezza del campione prelevato. Infine, riducendo al minimo il tempo di permanenza dell'ago nel rene, ha reso la biopsia più sicura e ha diminuito l'incidenza di complicanze post-bioptiche, consentendone comunque una più agevole diagnosi ed un accurato monitoraggio nel tempo.

\section{BIBLIOGRAFIA}

1

Iversen P, Brun C. Aspiration biopsy of the kidney. Am J Med 1951; 11: 324.

Madaio MP. Renal biopsy (Nephrology forum). Kidney Int 1990; 38: 529.

Kark RM, Muehrcke RC. Biopsy of the kidney in prone position. Lancet 1954; 1: 1047.

Welt L. Renal biopsy. JAMA 1968; 205: 220.

Haddad JK, Mani RL. Percutaneous renal biopsy. An improved method using television monitoring and high-dose infusion pyelography. Arch Intern Med 1957; 19: 157.

Telfer N, Ackroy DAE, Stock SL. Radioisotopic localization for renal biopsy. Lancet $1964 ; 1: 132$.

Colgan JR, Bischel M, Morrown JW. Retrograde catheter localization for percutaneous renal biopsy. JAMA 1971; 217: 824.

Rao K. Percutaneous renal transplant biopsy under CAT scanner guidance. Nephron 1984; 38: 273.

Birnholz JC, Kasinath BS, Corwin HL. An improved technique for ultrasound guided percutaneous renal biopsy. Kidney Int 1985; $27: 80$.

Yoshimoto M, Fujisawa S, Judo M. Percutaneous renal biopsy well-visualized by orthogonal ultrasound application using linear scanning. Clin Nephrol 1988; 30: 106.

11 Wiseman DA, Hawkins R, Numerow LM, Taub KJ. Percutaneous renal biopsy utilizing real time, ultrasonic guidance and a semiautomated biopsy device.
Kidney Int 1990; 38: 347.

Campobasso N, Pannarale G, Rizzi R, Schena FP. Biopsia renale ecoguidata con sonda settoriale: esperienza clinica su 126 biopsie consecutive. Atti del XXXII Congresso Nazionale della Società Italiana di Nefrologia. Monduzzi ed 1991: 605.

13 Donovan KL, Thomas DM, Wheeler DC, MacDougall IC, Williams JD. Experience with a new method for percutaneous renal biopsy. Nephrol Dial Transplant 1991; 6: 731.

14 Burstein DM, Schwartz MM, Korbet SM. Percutaneous renal biopsy with the use of realtime ultrasound. Am J Nephrol 1991; 11: 195.

15 Wickre CG, Golper TA. Complications of percutaneous renal biopsy. Ann Intern Med 1988; 108: 301.

16 Ginsburg JC, Fransman SL, Singer MA, Cohanim M, Morrin PAF. Use of computerized tomography (CT) to evaluate bleeding after renal biopsy. Nephron 1980; 26: 240 .

17 Rosenbaum R, Hoffsten PE, Stanley RJ, Klahr S. Use of computerized tomography to diagnose complications of percutaneous renal biopsy. Kidney Int 1978; 14: 87.

18 Alter AJ, Zimmerman S, Kirachaiwanich. Computerized tomographic assessment of retroperitoneal hemorrage after percutaneous renal biopsy. Arch Intern Med 1980; 140: 1323. 